\title{
In Vivo Chromatic Aberration in Eyes Implanted With Intraocular Lenses
}

\author{
Pablo Pérez-Merino, ${ }^{1}$ Carlos Dorronsoro, ${ }^{1}$ Lourdes Llorente, ${ }^{1}$ Sonia Durán, ${ }^{2}$ \\ Ignacio Jiménez-Alfaro, ${ }^{2}$ and Susana $\operatorname{Marcos}^{1}$ \\ ${ }^{1}$ Instituto de Óptica "Daza de Valdés," Consejo Superior de Investigaciones Científicas (CSIC), Madrid, Spain \\ ${ }^{2}$ Fundación Jiménez Díaz, Madrid, Spain
}

Correspondence: Susana Marcos, Instituto de Óptica "Daza de Valdés," Consejo Superior de Investigaciones Científicas, C/Serrano, 121, 28006 Madrid, Spain;

susana@io.cfmac.csic.es.

Submitted: February 21, 2013 Accepted: March 7, 2013

Citation: Pérez-Merino P, Dorronsoro C, Llorente L, Durán S, Jiménez-Alfaro I, Marcos S. In vivo chromatic aberration in eyes implanted with intraocular lenses. Invest Ophthalmol Vis Sci. 2013;54:2654-2661. DOI:10. $1167 /$ iovs.13-11912
Purpose. To measure in vivo and objectively the monochromatic aberrations at different wavelengths, and the chromatic difference of focus between green and infrared wavelengths in eyes implanted with two models of intraocular lenses (IOL).

Methods. Eighteen eyes participated in this study: nine implanted with Tecnis ZB99 1-Piece acrylic IOL and nine implanted with AcrySof SN60WF IOL. A custom-developed laser ray tracing (LRT) aberrometer was used to measure the optical aberrations, at $532 \mathrm{~nm}$ and 785 $\mathrm{nm}$ wavelengths. The monochromatic wave aberrations were described using a fifth-order Zernike polynomial expansion. The chromatic difference of focus was estimated as the difference between the equivalent spherical errors corresponding to each wavelength.

Results. Wave aberration measurements were highly reproducible. Except for the defocus term, no significant differences in high order aberrations (HOA) were found between wavelengths. The average chromatic difference of focus was $0.46 \pm 0.15$ diopters (D) in the Tecnis group, and $0.75 \pm 0.12 \mathrm{D}$ in the AcrySof group, and the difference was statistically significant $(P<0.05)$. Chromatic difference of focus in the AcrySof group was not statistically significantly different from the Longitudinal chromatic aberration (LCA) previously reported in a phakic population $(0.78 \pm 0.16 \mathrm{D})$. The impact of LCA on retinal image quality (measured in terms of Strehl ratio) was drastically reduced when considering HOA and astigmatism in comparison with a diffraction-limited eye, yielding the differences in retinal image quality between Tecnis and AcrySof IOLs not significant.

Conclusions. LRT aberrometry at different wavelengths is a reproducible technique to evaluate the chromatic difference of focus objectively in eyes implanted with IOLs. Replacement of the crystalline lens by the IOL did not increase chromatic difference of focus above that of phakic eyes in any of the groups. The AcrySof group showed chromatic difference of focus values very similar to physiological values in young eyes.

Keywords: longitudinal chromatic aberration, intraocular lens, aberrometry
$\mathbf{R}$ etinal image quality is determined by the combined optical aberrations of the cornea and crystalline lens, pupil size, and intraocular scattering. In eyes with cataract, scattering by the opacified lens causes a major decrease in image quality. Upon replacement of the crystalline lens by an artificial lens, the source of scattering is eliminated and refractive errors are generally well corrected. State-of-the art aspheric designs also aim at compensating the spherical aberration of the cornea. ${ }^{1-6}$ In natural conditions, both monochromatic and chromatic aberrations play a role in determining retinal image quality. ${ }^{7-18}$ In fact, it has been shown that interactions between monochromatic and chromatic aberrations occur, and that the presence of monochromatic aberrations partly attenuates the optical degradation produced by the longitudinal chromatic aberration (LCA), or vice versa. ${ }^{14,18}$ Modifications in either the monochromatic or chromatic aberration component may alter this compensatory effect found in the natural eye.

LCA in the eye is determined by dispersion of light in the intraocular media and in the crystalline lens. ${ }^{7,8}$ Unlike transverse chromatic aberration (TCA), which shows a high intersubject variability, LCA is less variable across subjects, and seems to remain fairly constant with age..$^{7-10,13,14,17,18}$
The replacement of the crystalline lens by an intraocular lens (IOL) modifies the chromatic dispersion properties of the eye, according to the dispersion properties of the IOL material (defined by the Abbe number). Reports of the Abbe number of different IOL materials range between 35 to 60 (37 for the Alcon acrylic, 55 for the Tecnis acrylic) ${ }^{20}$ In principle, the higher the Abbe number, the lower the LCA. This role of the IOL material on the chromatic difference of focus of the pseudophakic eye has been already acknowledged, ${ }^{19-21}$ and it has led to proposals for IOL designs aiming at correcting the chromatic aberration of the eye. ${ }^{22,23}$ This has also prompted studies on the expected performance of eyes corrected for LCA both computationally from real aberration measurements ${ }^{13,16}$ or psychophysically. ${ }^{15}$

The chromatic aberrations of the phakic eye have been studied widely, and numerous studies report experimental measurements (psychophysical or objective) of the LCA in normal phakic eyes. ${ }^{7-16}$ However, most estimates of the LCA in pseudophakic eyes are based on computer simulations, using data for the Abbe number of the lens material ${ }^{19}$ or on bench measurements of the isolated IOLs. ${ }^{24}$ To our knowledge, only one study measured the LCA in vivo (between 500 and $650 \mathrm{~nm}$ ) 
in pseudophakic eyes implanted with PMMA and acrylic IOLs, ${ }^{20}$ using a modified chromoretinoscopy ${ }^{25}$ system.

In a previous study, we reported the measurement of chromatic difference of focus based on aberrometry at two different wavelengths. ${ }^{16}$ In particular, the use of laser ray tracing (LRT) or Hartmann-Shack aberrometry using different illumination may allow us rapid and reliable measurement of LCA in phakic subjects. Aberrometry provides, in addition, monochromatic high-order aberrations (HOA) measurements. This allows testing the correction/induction of HOA for a given IOL design, and ultimately, estimating the polychromatic image quality in the pseudophakic eye. ${ }^{17}$

In the present study, we measured monochromatic aberrations in both $532 \mathrm{~nm}$ (green) and $785 \mathrm{~nm}$ (IR) wavelengths in patients implanted with AcrySof IQ SN60WF (Alcon Research Labs, Fort Worth, TX) and with Tecnis ZCB00 1-Piece (Abbot Medical Optics Inc., Santa Ana, CA) IOLs. We estimated the LCA as the chromatic difference of focus between the equivalent spherical error corresponding to each wavelength, by using a previously described and validated aberrometry-based methodology. To our knowledge, this is the first report of both monochromatic and chromatic aberrations in pseudophakic patients, as well as the first report in vivo of the chromatic difference of focus of two of the most widespread IOL materials.

\section{Materials AND Methods}

\section{Patients, Surgery, and IOLs}

Eighteen eyes from eighteen patients participated in the study, nine implanted with the Tecnis ZCB00 1-Piece (Abbot Medical Optics Inc.), and nine implanted with the AcrySof IQ SN60WF (Alcon Research Labs). Both IOLs are monofocal, acrylic and aspheric, but they differ in the specific optical design and material. Table 1 shows the age and refractive profiles of the two groups of patients.

Selection criteria of the patients included good general health, no ocular pathology, and no complications during surgery. All enrolled patients provided informed consent. The protocols had beenapprovedbytheInstitutionalReviewBoard,andmetthetenets of the Declaration of Helsinki. Patients received a comprehensive ophthalmic evaluation at the hospital (Fundación Jiménez Díaz, Madrid, Spain) prior to enrollment in the study and surgery. The examination included uncorrected and best-corrected visual acuity, biomicroscopy, keratometry, corneal topography, tonometry, and indirect ophthalmoscopy. Axial length and anterior chamber depth were measured with optical biometry (IOL Master 500; Carl Zeiss Meditec AG, Jena, Germany). The IOL power was calculated with the SRK-T formula, always selecting the closest value to emmetropia.

Postoperative evaluations at the hospital were conducted at 1 day, 1 week, 1 month, and 3 months after surgery, and included uncorrected and best-corrected visual acuity, autorefractometry, manifest refraction, biomicroscopy, keratometry, tonometry, and indirect ophthalmoscopy.

All procedures were performed by the same surgeon (SD) on an outpatient basis under topical anesthesia. A 2.2- $\mathrm{mm}$ corneal incision and a paracentesis were performed with a surgical knife. A 6.0-mm continuous curvilinear capsulorhexis

Table 1. Age and Refractive Profiles of the Tecnis and AcrySof Groups

\begin{tabular}{lcc}
\hline & $\begin{array}{c}\text { Tecnis Group, } \\
\boldsymbol{n}=\mathbf{9}\end{array}$ & $\begin{array}{c}\text { AcrySof Group, } \\
\boldsymbol{n}=\mathbf{9}\end{array}$ \\
\hline Age, mean \pm SD & $73.4 \pm 10.9$ & $74.3 \pm 7.2$ \\
IOL power, mean \pm SD & $21.2 \pm 0.8$ & $22.0 \pm 1.6$ \\
\hline
\end{tabular}

was made under viscoelastic material. Phacoemulsification of the lens was performed with a commercial microsurgical system (Millennium Microsurgical System; Bausch \& Lomb, Rochester, NY). After removing cortical material, the surgeon proceeded to clean the anterior and posterior capsules with the automatic I-A straight tip. Both foldable posterior chamber lenses were implanted using the Monarch III injector (Alcon Research Labs) through the $2.2 \mathrm{~mm}$ incision. Once the viscoelastic material was removed, the incision was closed by hydration without sutures. Postoperatively, patients were treated with a combination of antibiotic and corticosteroid drops (dexamethasone plus tobramycin) for 4 weeks.

\section{Laser Ray Tracing}

Total wave aberrations were measured at 3 months after surgery using a custom LRT optical system. The technique has been described in detail in previous studies. ${ }^{16,26,27}$ Illumination was provided by two collinear laser diodes (laser-diode pumped green He-Ne laser at $532 \mathrm{~nm}$ [Brimrose, Baltimore, MD]), and an IR laser diode at $785 \mathrm{~nm}$ (Schäfter + Kirchhoff, Hamburg, Germany). The beam samples the pupil sequentially, and the sampling pattern can be configured via custom-designed software. A sampling pattern consisting of 37 entry positions arranged in a hexagonal configuration was used for this study. The sampling pattern was adjusted by custom-designed software to fit the pupil of the patient's eye, which ranged from 4 to $6 \mathrm{~mm}$ after inducing mydriasis with 1 drop of tropicamide $1 \%$. Measurements were done in a dark room with the subject fixating at a foveal target. The eye was monitored during measurements with a charge-coupled device (CCD) camera (model XC-55; Sony Corp., Tokyo, Japan) conjugate to the pupil in order to ensure a correct alignment between the pupil center and the optical axis of the setup. The patient's subjective refraction was corrected with a Badal system. Maximum energy exposure was $4.1 \mu \mathrm{W}$ at $532 \mathrm{~nm}$ and $6.8 \mu \mathrm{W}$ at $785 \mathrm{~nm}$. A highsensitivity CCD camera (Retiga 2000R; QImaging, Surray, BC, Canada) recorded the retinal aerial images corresponding to each entry pupil beam. Each set of measurements consisted of five runs for green and five for IR wavelengths under the same conditions, and the results presented are the average of the corresponding five repeated measurements.

\section{Data Analysis}

Ray aberrations were estimated from the deviations of the centroids of the retinal images corresponding to each entry pupil location from the reference (chief ray), using commercial technical computing software (MATLAB; MathWorks, Inc., Natick, MA). These deviations are proportional to the local derivatives of the monochromatic wave aberrations. The monochromatic wave aberration was described with Zernike polynomials up to seventh order. The spherical error for each wavelength was estimated considering different definitions for spherical equivalent error (M). ${ }^{28,29}$

$$
\begin{aligned}
& M=\frac{-4 \sqrt{3} C_{2}^{0}}{R^{2}} \\
& M=\frac{-4 \sqrt{3} C_{2}^{0}+12 \sqrt{5} C_{4}^{0}-24 \sqrt{7} C_{6}^{0}}{R^{2}} \\
& J_{0}=\frac{-2 \sqrt{6} C_{2}^{2}}{R^{2}} ; J_{45}=\frac{-2 \sqrt{6} C_{2}^{-2}}{R^{2}} ; C=-2 \sqrt{J_{0}^{2}+J_{45}^{2}} ; \\
& M=\left(\frac{-4 \sqrt{3} C_{2}^{0}}{R^{2}}-C\right) / 2
\end{aligned}
$$


The chromatic difference of focus was then estimated as the difference between the spherical equivalent error obtained for green and IR wavelengths, as described in an earlier study from our laboratory on phakic subjects. ${ }^{16}$ The analysis was done for a 4-mm effective pupil diameter for all subjects. Point spread functions (PSFs) were computed using standard Fourier optics for the same pupil diameter. Image quality was analyzed in terms of Strehl ratio, defined as the maximum of the PSF relative to the maximum of the diffraction-limited PSF. Strehl ratio is an appropriate optical quality metric in nonhighly aberrated optical systems, and in the absence of large amounts of defocus, as in this study. ${ }^{30}$ Besides, through-focus analysis of Strehl ratio has been shown to allow accurate estimates of the best subjective focus. ${ }^{31,32}$ PSFs in green were computed at best focus (that maximizing Strehl ratio); PSFs in IR were computed assuming the chromatic difference of focus in the defocus term. The effect of the chromatic difference of focus was evaluated on average PSFs for each group (Tecnis or AcrySof). Average PSFs in focus and defocused by the chromatic difference of focus were computed by averaging individual PSFs in each condition, assuming no aberrations (diffractionlimited ideal case); the measured HOA only; and HOA and astigmatism (from IR aberration data). All computations were performed for 4-mm pupils. Univariate analysis (independent samples Student's $t$-test) was used to evaluate the differences in chromatic difference of focus between green and IR, as well as in monochromatic aberrations and optical quality between the two groups implanted with the IOLs.

\section{Results}

\section{Monochromatic Aberrations}

Figure 1 shows the Zernike coefficients, and the corresponding wave aberration maps (excluding tilt, defocus, and astigmatism), for two representative subjects from the Tecnis and AcrySof groups, respectively. Repeated wave aberration measurements were highly reproducible within each subject. The RMS standard deviation for HOA for repeated measurements was $0.04 \mu \mathrm{m}$ (averaged across subjects). The standard deviation for the defocus Zernike term for repeated measurements was $0.06 \mu \mathrm{m}$ (averaged across subjects).

The average value for defocus $\left(\mathrm{Z}_{2}{ }^{0}\right)$; astigmatism-term $\left(\mathrm{Z}_{2}{ }^{2}\right.$ and $\left.\mathrm{Z}_{2}{ }^{-2}\right)$; spherical aberration $\left(\mathrm{Z}_{4}^{0}\right)$; coma-like term $\left(\mathrm{Z}_{3}{ }^{1}\right.$ and $\mathrm{Z}_{3}{ }^{-1}$ ); and the RMS for HOAs for the Tecnis as well as for the AcrySof group are summarized in Table 2, for both green and IR wavelengths. For both IOL groups, the defocus term shows significant differences across wavelengths $(P<0.05)$. However, the levels of astigmatism, coma, spherical aberration, and total HOAs are very similar between Tecnis and AcrySof IOLs.

\section{Chromatic Difference of Focus}

As expected, the defocus term was significantly different across wavelengths in both Tecnis and AcrySof groups (see Table 2). Figure 2 shows the chromatic difference of focus (estimating LCA) expressed in diopters (D) between green and IR wavelengths in both groups, using the different definitions for spherical equivalent error (M). The average chromatic difference of focus (from Equation 1) in patients implanted with Tecnis was $0.46 \pm 0.15 \mathrm{D}$ and in patients implanted with AcrySof was $0.76 \pm 0.12 \mathrm{D}$, between 532 (green) and $785 \mathrm{~nm}$ (IR). The chromatic difference of focus of a phakic population $(0.78 \pm 0.16$ D) from an earlier study using the same instrument is also used for comparison with our results. ${ }^{17}$ The difference in LCA between the Tecnis and the phakic population of our previous study (nine subjects) was statistically significant different $(P<0.05)$, whereas there was no statistically significant differences between the AcrySof and phakic groups.

\section{Effect of Chromatic Difference of Focus on Retinal Image Quality}

Figure 3 shows simulated PSFs from monochromatic aberration measurements at green and IR wavelengths for all subjects, including astigmatism and HOAs. PSFs varied significantly across subjects for both the AcrySof IOLs and Tecnis IOLs, with some subjects showing markedly asymmetric PSFs (dominated by coma and/or astigmatism) while others showing closer to diffraction-limited intensity distribution. The effect of the defocus produced by the chromatic difference of focus on the IR PSF appears more dependent on the amount of present astigmatism and HOA than on the lens type defocus produced a larger degradation on the highest quality PSFs (more so in eyes implanted with the AcrySof IOL). For example, Strehl ratio changed from 0.16 (green) to 0.007 (IR) in $\mathrm{S} \# 2$, and from 0.14 (green) to 0.007 (IR) in $\mathrm{S} \# 13$, in the presence of chromatic defocus. On the other hand, the chromatic defocus produced a relatively lower degradation in higher aberrated eyes (i.e., Strehl ratios from 0.021 [green] to 0.020 [IR] in $\mathrm{S} \# 9$ ), and from 0.018 (green) to 0.010 (IR) in $S \# 10$. In eyes with astigmatism, chromatic defocus moved the best focus (i.e., the focus that maximized Strehl ratio) along the Sturm interval.

Figure 4 illustrates the effective impact of the chromatic difference of focus on the image quality for both IOLs, in terms of PSFs (Figs. $4 \mathrm{a}-\mathrm{c}$ ) and Strehl ratios (Figs. 4d-f), averaged across subjects in each group, and for 4-mm diameter pupils. The PSFs (all based on IR measurements) are shown in best focus (upper panels) and defocused by the corresponding chromatic difference of focus (lower panels), for both Tecnis and AcrySof. Three conditions were tested: assuming diffraction-limited optics (i.e., full correction of astigmatism and HOAs); considering the measured HOA aberrations present (excluding astigmatism); and considering both measured HOA and astigmatism. In the absence of HOA (Fig. 4d), Strehl ratio decreased from 1 (in focus) to 0.08 (defocused) in eyes implanted with Tecnis, and to 0.01 in eyes implanted with AcrySof. However, the presence of real HOA and astigmatism diminished dramatically the impact of chromatic difference of focus on retinal image quality. HOA decrease image quality at best focus with respect to diffraction-limit. However chromatic defocus attenuated retinal image quality in real eyes with HOA to a much lesser extent than in diffraction limited eyes. Average Strehl ratio was $0.15 \mathrm{D}$ in both Tecnis and Acrysof eyes without chromatic defocus, and $0.09 \pm 0.05$ for Tecnis and $0.05 \pm 0.03$ for Acrysof, with chromatic defocus (Fig. 4e). The presence of the subjects' astigmatism (Fig. 4f) further degraded image quality in focus (Strehl ratio of 0.08 , both for Tecnis and AcrySof) and further attenuated the impact of chromatic defocus (Strehl ratios of $0.03 \pm 0.02$ for Tecnis and $0.02 \pm$ 0.01 for AcrySof).

When evaluated in terms of retinal image quality metrics (Strehl), we did not find statistical differences between in focus image quality of eyes implanted with Tecnis or AcrySof (HOA only, and HOA and astigmatism; $P>0.5$ ). Also, we did not find differences in the chromatic defocused conditions between the two lenses (HOA only, $P=0.08$; HOA and astigmatism, $P>$ $0.5)$. Optical quality in focus and with chromatic defocus were statistically significant different with astigmatism $(P<0.05)$ in both IOLs, and without considering astigmatism in AcrySof $(P$ $<0.05$ ) but not in Tecnis. 


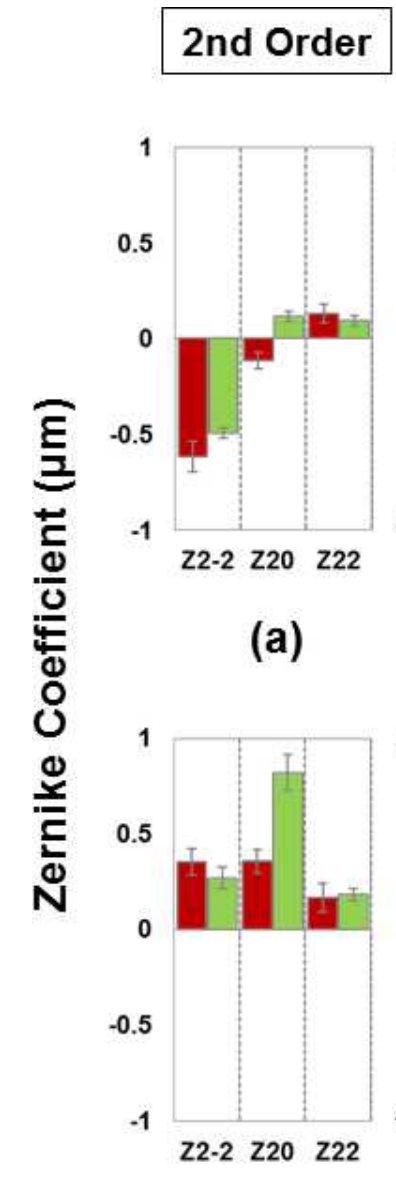

(d)

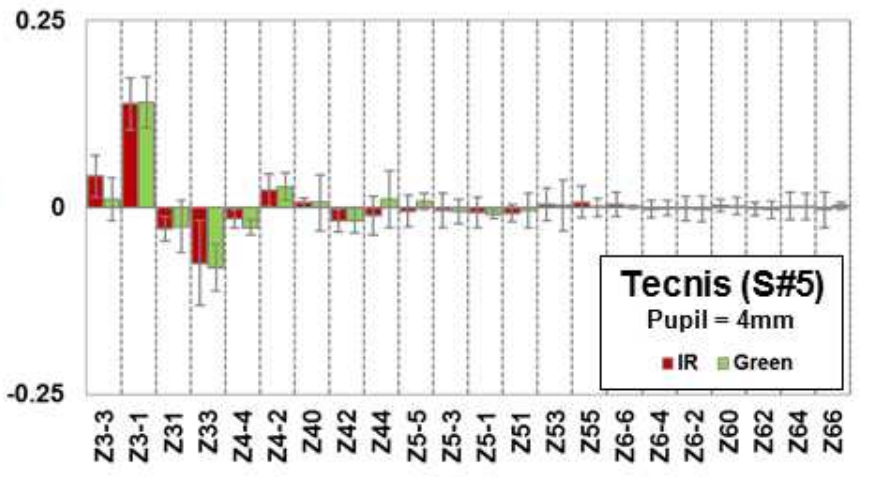

(b)

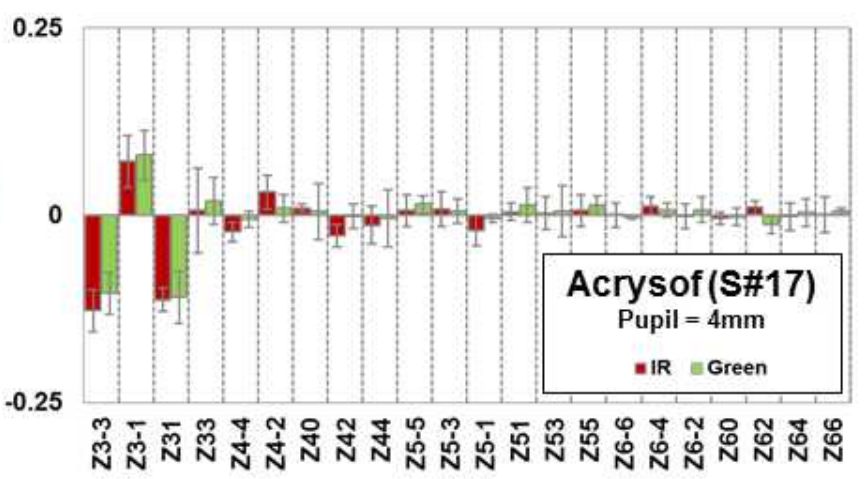

(e)
Wave aberration maps

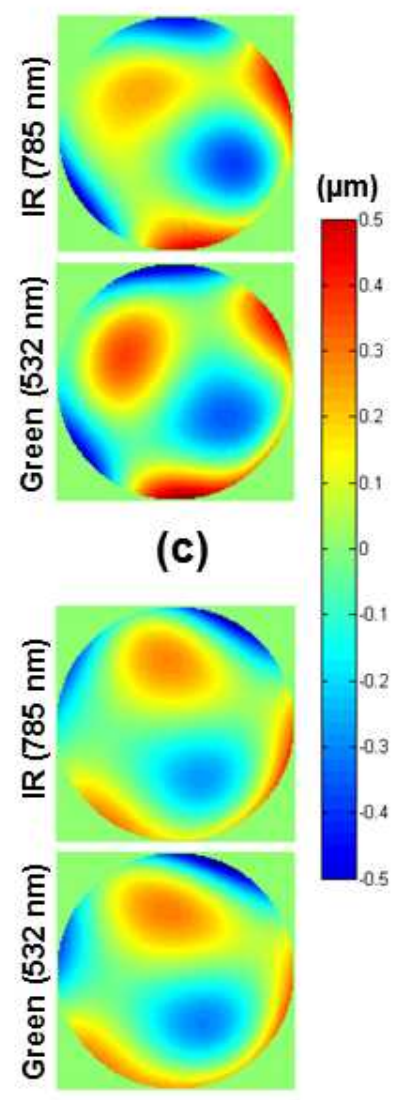

(f)

Figure 1. Examples of Zernike coefficients and wave aberration maps measured in green and IR. (a) Second-order and (b) Higher-order Zernike coefficients (averaged across 5 repeated measurements) for a representative eye implanted with Tecnis (S\#5), for IR (785 $\mathrm{nm}$, red column) and green $(532 \mathrm{~nm}$, green column) illumination. (c) Wave aberration maps (calculated from average Zernike coefficients excluding tilt, defocus, and astigmatism) for IR (785 nm, upper) and green (532 nm, lower; Tecnis [S\#5]). (d) Second-order and (e) Higher-order Zernike coefficients (averaged across five repeated measurements) for a representative eye implanted with AcrySof (S\#17), for IR ( $785 \mathrm{~nm}$, red column) and green (532 nm, green column). (f) Wave aberration maps (calculated from average Zernike coefficients excluding tilt, defocus, and astigmatism) for IR (785 nm, upper) and green $(532 \mathrm{~nm}$, lower), respectively (AcrySof [S\#17]).

\section{Discussion}

We have shown that LRT aberrometry using different wavelengths is a reproducible technique to measure monochromatic aberrations, as well as chromatic difference of focus in vivo in eyes implanted with different IOLs. Intrasubject repeatability in chromatic difference of focus estimates was high, and the intersubject variability in LCA $(0.15 \mathrm{D}$ and $0.12 \mathrm{D}$, for Tecnis and AcrySof, respectively) was similar or even smaller to that of values reported in previous studies in phakic

TABLE 2. Effective Defocus and RMS Values for Spherical, Astigmatism, Coma, and Total HOAs in Tecnis and AcrySof Groups

\begin{tabular}{|c|c|c|c|c|c|}
\hline & Defocus, $D^{*}$ & Astigmatism, $\mu \mathrm{m} \dagger$ & Spherical, $\mu \mathrm{m} \ddagger$ & Coma, $\mu \mathrm{m} \S$ & Total HOAs, $\mu \mathrm{m} \mid$ \\
\hline \multicolumn{6}{|l|}{$532 \mathrm{~nm}$} \\
\hline Tecnis & $-0.14 \pm 0.15$ & $0.36 \pm 0.26$ & $0.01 \pm 0.03$ & $0.14 \pm 0.07$ & $0.21 \pm 0.08$ \\
\hline AcrySof & $-0.28 \pm 0.27$ & $0.46 \pm 0.24$ & $0.02 \pm 0.03$ & $0.09 \pm 0.03$ & $0.17 \pm 0.04$ \\
\hline \multicolumn{6}{|l|}{$785 \mathrm{~nm}$} \\
\hline Tecnis & $0.30 \pm 0.17$ & $0.39 \pm 0.30$ & $0.01 \pm 0.03$ & $0.13 \pm 0.09$ & $0.22 \pm 0.11$ \\
\hline AcrySof & $0.41 \pm 0.24$ & $0.49 \pm 0.18$ & $0.02 \pm 0.02$ & $0.09 \pm 0.03$ & $0.18 \pm 0.04$ \\
\hline
\end{tabular}

Data are shown as mean \pm standard deviation.

* $\mathrm{Z}_{2}{ }^{\circ}$.

$+\mathrm{Z}_{2}{ }^{2}$ and $\mathrm{Z}_{2}^{-2}$.

$\neq Z_{4}{ }^{\circ}$.

$\int \mathrm{Z}_{3}{ }^{1}$ and $\mathrm{Z}_{3}{ }^{-1}$

$\|$ Mean $\pm S D$. 


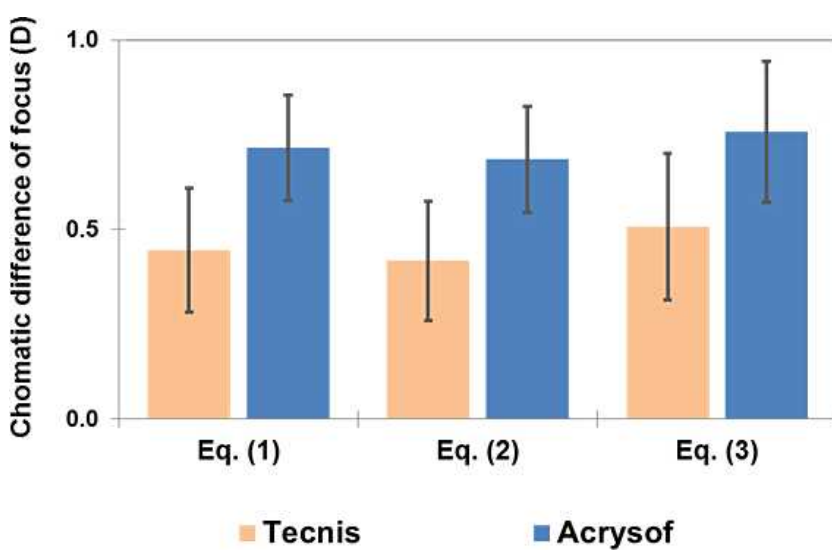

Figure 2. Chromatic difference of focus for Tecnis and AcrySof between 532 and $785 \mathrm{~nm}$ wavelengths. Equation 1, Equation 2, and Equation 3 correspond to different definitions for spherical equivalent error. $^{28,29}$

eyes using both objective $\left(\begin{array}{lll}0.29 & \mathrm{D}\end{array}\right)^{16}$ and psychophysical techniques $(0.16 \mathrm{D}),{ }^{13}$ or those from the only report in pseudophakic eyes (0.18 D and 0.22 D, for PMMA and AcrySof, respectively). ${ }^{20}$

Our in vivo measurements of chromatic difference of focus (0.46 D and $0.76 \mathrm{D}$ in eyes implanted with Tecnis and AcrySof IOLs, respectively) are, in general, consistent with theoretical predictions using computer eye models and the nominal/measured material Abbe numbers in our range of wavelengths $(0.35 \mathrm{D}$ and $0.65 \mathrm{D}$, for Tecnis and AcrySof, respectively). ${ }^{19}$ The reported chromatic difference of focus is lower than the longitudinal chromatic aberration for the entire visible wavelength range. Our measurements are restricted to the longer wavelength part of the spectrum, although the impact of the short wavelength range on vision is relatively minor, due to the reduced density of blue cones, and the important absorption of light in the macular pigment and in the IOL yellow filters (AcrySof). In addition, doublepass based measurements of LCA tend to be lower than psychophysical measurements of LCA. ${ }^{7,12}$ In contrast, chromoretinoscopy measurements performed on patients implanted with PMMA and AcrySof IOLs (the only previous report of LCA measured in vivo on pseudophakic patients) ${ }^{20}$ overestimate LCA with respect to theoretical computations. In comparison with chromoretinoscopy experimental measurements, theoretical estimates of LCA (between 500 and 640 $\mathrm{nm}$ ) in eyes implanted with AcrySof IOLs were on average $0.22 \mathrm{D}$ lower (modeling by Nagata et $a{ }^{20}{ }^{20}$ ) or $0.4 \mathrm{D}$ (modeling by Zhao et al. ${ }^{19}$ ). As expected, we found a consistently lower LCA in eyes implanted with Tecnis IOLs (reported Abbe number = 55) than in eyes implanted with AcrySof (reported Abbe number $=37$ ). A comparison with the LCA of a group of phakic patients ${ }^{16}$ measured with the same instrument revealed that the Tecnis group had a significantly lower LCA than the natural crystalline lens, but the differences between the AcrySof and the phakic subjects were not statistically significant.

The correction of the LCA in the eye has been long been debated and proposals of LCA-correcting IOLs have been made, mostly in the form of diffractive elements. ${ }^{22,23}$ While the monochromatic MTF of the eye clearly exceeds the polychromatic $\mathrm{MTF}^{13,14}$ and there is evidence that, in the absence of both chromatic and monochromatic aberrations, visual performance exceeds that with noncorrected chromatic aberrations, ${ }^{15,18}$ correction of LCA alone has not yielded remarkable vision correction. ${ }^{33}$ Reasons for this relatively low

\section{Tecnis (PSFs)}

\section{Green} $(532 \mathrm{~nm})$

IR (785 nm)

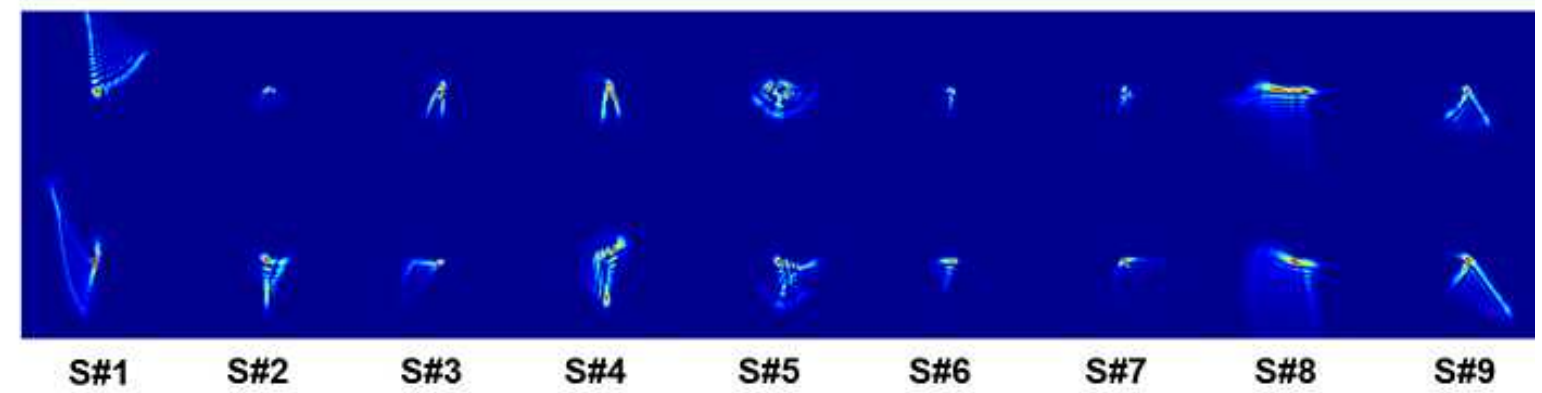

\section{Acrysof (PSFs)}

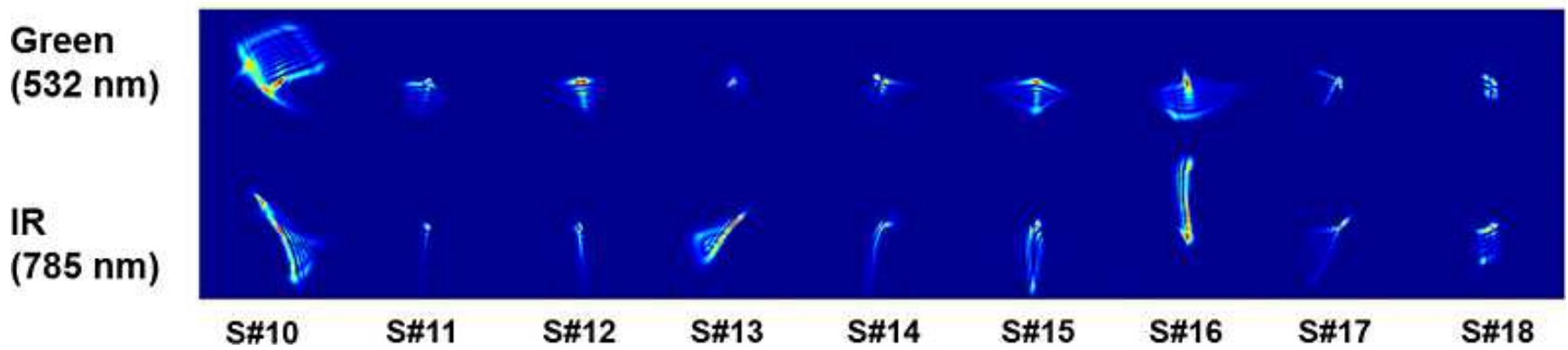

Figure 3. Simulated PSFs from the wave aberrations corresponding to all subjects of the study (pupil size $=4 \mathrm{~mm}$ ), implanted with Tecnis and AcrySof) for all eyes in green (at the best focus) and IR (defocused by the chromatic difference of focus) wavelengths 


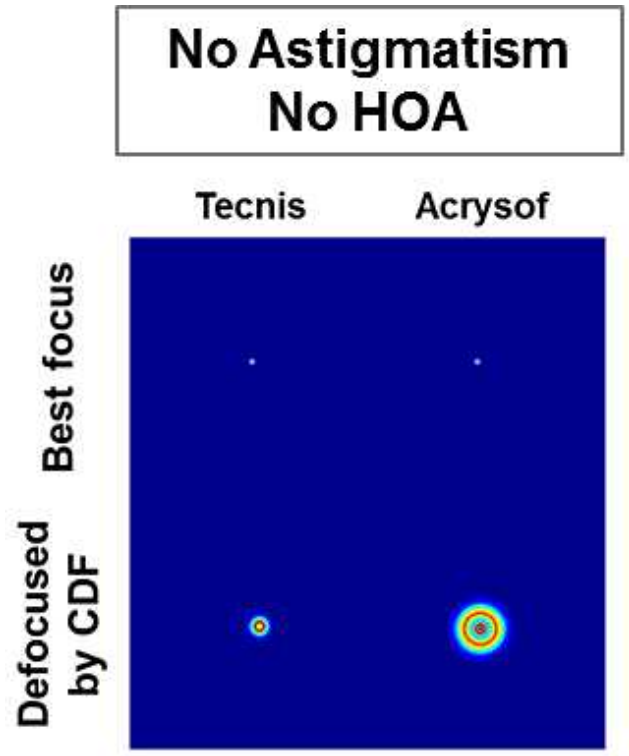

(a)

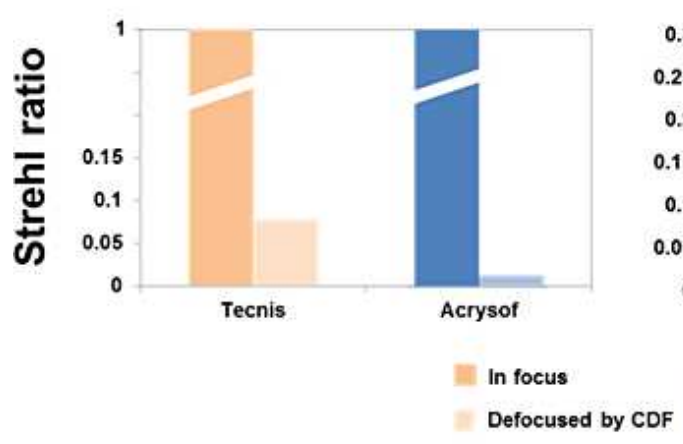

(d)
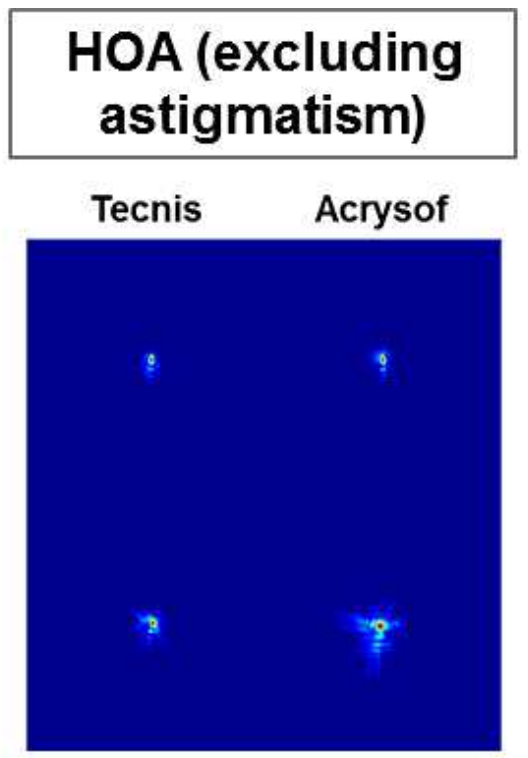

(b)

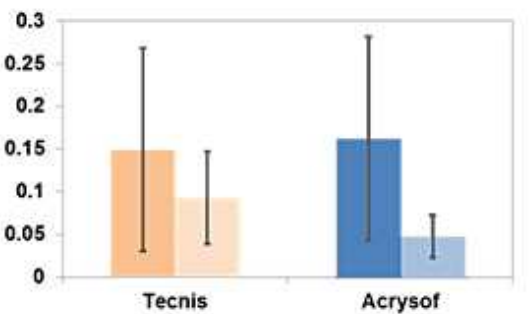

Tecnis
In focus

Defocused by CDF

\section{Astigmatism HOA}

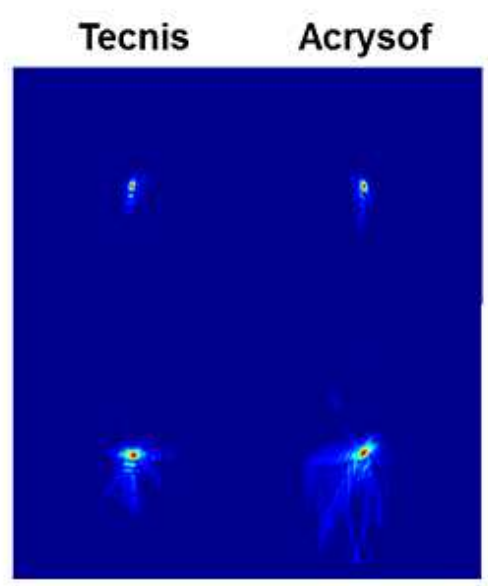

(c)

Figure 4. Simulated effect of chromatic difference of focus on the PSFs and Strehl ratio. (a) Average PSF excluding astigmatism and HOAs at best focus - that is, diffraction-limited (top) - and defocused by the measured chromatic difference of focus G-IR (bottom) of the Tecnis (left) and AcrySof (right) IOLs. (b) Average PSF with HOAs, excluding astigmatism at best focus (top) and defocused by the chromatic difference of focus (bottom) for both groups. (c) Average PSF with HOAs and astigmatism at best focus (top) and defocused by the chromatic difference of focus (button) for both groups. Average Strehl ratios in eyes implanted with Tecnis and AcrySof, in focus and defocused by the chromatic difference of focus (d) for a theoretical diffraction-limited eye, (e) for HOAs without astigmatism, and (f) for HOAs and astigmatism. CDF, Chromatic difference of focus. Error bars (standard deviations across patients).

benefit of correcting LCA include the presence of TCA, ${ }^{13,14,17,18}$ and the fact that monochromatic aberrations and LCA interact favorably in eyes with physiological amounts of aberrations. McLellan et al. ${ }^{14}$ and Ravikumar et al. ${ }^{18}$ reported that, in fact, the presence of monochromatic aberrations attenuated the degrading effect of the chromatic aberration, particularly for shorter wavelengths, in contrast with a diffraction-limited eye where chromatic defocus produced large differences in the MTF across wavelengths. As the IOLs become more sophisticated in design (ultimately aiming at correcting the $\mathrm{HOA}$ of the individual eye), $, 4,6,22,23$ the correction of LCA may become more relevant. Both IOLs of the study had aspheric surfaces, and aimed by design at correcting (or at least reducing) the positive spherical aberration of the average cornea, ${ }^{3,6}$ similarly to the corneal/ internal balance of spherical aberration in the young eye. ${ }^{34,35}$ The measured HOA in the pseudophakic patients of the study (average RMS_HOA $=0.21 \pm 0.08 \mu \mathrm{m}$ [Tecnis] and $0.17 \pm$ $0.04 \mu \mathrm{m}$ [AcrySof], green light, 4-mm pupil) were of the order of magnitude of those found in a young population (average RMS_HOA $=0.70 \pm 0.11 \mu \mathrm{m}$, green light, $6.51-\mathrm{mm}$ pupil). ${ }^{16}$ Our simulations of the PSFs reveal the image quality degradation produced by the HOAs in the tested pseudophakic patients, which changed in shape and magnitude across eyes. According to the mentioned prior literature, physiological amounts of LCA are not greatly detrimental to retinal image quality, as a result of the positive interaction with the existing natural HOA. In fact, in most eyes, the interactions of HOA and astigmatism with chromatic defocus attenuated the impact of the chromatic difference of focus on the PSF, very much like the effect of pure defocus, having a lesser relative impact on image degradation in the presence of HOA and astigmatism than in a diffraction-limited eye. As seen in Figures 3 and 4 , the relative impact of the chromatic difference of focus in eyes with HOAs (with or without astigmatism), is much lower than that expected in a diffraction limited eye (Figs. 4a, 4d), with no significant differences in the degradation of the PSF with LCA between 
groups. Other functions that have been suggested to be helped by the presence of LCA include emmetropization and accommodation. ${ }^{36,37}$

Aberrometry-based measurements provide both HOA (and therefore an evaluation of the aberration correction/induction produced by the IOL design) and chromatic difference of focus. A full estimation of retinal image quality in vivo would require measurements at a higher number of wavelengths (covering the visible spectrum), and individual estimates of TCA. The former can be performed by adding multiple lasers of different wavelengths or a supercontinuum laser source. The latter would require an additional psychophysical or objective measurement of TCA. ${ }^{17,38,39}$

\section{Acknowledgments}

Supported by Ministerio de Educación y Ciencia, Spain (Grants FIS2008-02065, FIS2011-25637); EURHORCs-ESF EURYI-05-102-ES and European Research Council (ERC-2011-AdG-294099; SM); and by a Collaborative Agreement with Alcon Research Labs, Fort Worth, Texas.

Disclosure: P. Pérez-Merino, None; C. Dorronsoro, None; L. Llorente, None; S. Durán, None; I. Jiménez-Alfaro, None; S. Marcos, Alcon Research Labs (F)

Presented at the XXX Congress of the European Society of Cataract \& Refractive Surgeons, Milan, Italy, September 2012.

\section{References}

1. Holladay JT, Piers PA, Koranyi G, van der Mooren M, Norrby NES. A new intraocular lens design to reduce spherical aberration of pseudophakic eyes. J Refract Surg. 2002;18: 683-691.

2. Barbero S, Marcos S, Jimenez-Alfaro I. Optical aberrations of intraocular lenses measured in vivo and in vitro. J Opt Soc Am A. 2003;20:1841-1851.

3. Marcos S, Barbero S, Jimenez-Alfaro I. Optical quality and depth-of-field of eyes implanted with spherical and aspheric intraocular lenses. J Refract Surg. 2005;21:223-235.

4. Tabernero J, Piers P, Benito A, Redondo M, Artal P. Predicting the optical performance of eyes implanted with IOLs to correct spherical aberrations. Invest Ophthalmol Vis Sci. 2006;47:4651-4658.

5. Franchini A. Compromise between spherical and chromatic aberration and depth of focus in aspheric intraocular lenses. $J$ Cataract Refract Surg. 2007;33:497-509.

6. Piers PA, Weeber HA, Artal P, Norrby S. Theoretical comparison of aberration-correcting customized and aspheric intraocular lenses. J Refract Surg. 2007;23:374-384.

7. Charman WN, Jennings JAM. Objective measurements of the longitudinal aberration of the human eye. Vision Res. 1976;16: 999-1005.

8. Howarth PA, Bradley A. The longitudinal chromatic aberration of the human eye, and its correction. Vision Res. 1986;26:361366.

9. Howarth PA, Zhang XX, Bradley A, Still DL, Thibos LN. Does the chromatic aberration of the eye vary with age? J Opt Soc Am A. 1988;5:2087-2092.

10. Thibos LN, Bradley A, Still DL, Zhang X, Howarth PA. Theory and measurement of the ocular chromatic aberration on monocular visual performance. Vision Res. 1990;30:33-49.

11. Thibos LN, Bradley A, Zhang X. Effect of ocular chromatic aberration on monocular visual performance. Optom Vis Sci. 1991;68:599-607.

12. Rynders M, Lidkea B, Chisholm W, Thibos LN. Statistical distribution of foveal transverse chromatic aberration, pupil centration, and angle psi in a population of young adult eyes. $J$ Opt Soc Am A. 1995;12:2348-2357.

13. Marcos S, Burns SA, Moreno-Barriuso E, Navarro R. A new approach to the study of ocular chromatic aberrations. Vision Res. 1999;39:4309-4323.

14. McLellan JS, Marcos S, Prieto PM, Burns SA. Imperfect optics may be the eye's defence against chromatic blur. Nature. 2002; $417: 174-176$.

15. Yoon GY, Williams DR. Visual performance after correcting the monochromatic and chromatic aberrations of the eye. $J O p t$ Soc Am A. 2002;19:266-275.

16. Llorente L, Diaz-Santana L, Lara-Salcedo D, Marcos S. Aberrations of the human eye in visible and near infrared illumination. Optom Vis Sci. 2003;80:26-35.

17. Marcos S, Burns A, Prieto PM, Navarro R, Baraibar B. Investigating sources of variability of monochromatic and transverse chromatic aberrations across eyes. Vision Res. 2001; $41: 3861-3871$.

18. Ravikumar S, Thibos LN, Bradley A. Calculation of retinal image quality for polychromatic light. J Opt Soc Am A. 2008; 25:2395-2407.

19. Zhao H, Mainster MA. The effect of chromatic dispersion on pseudophakic optical performance. Br J Ophthalmol. 2007; 91:1225-1229.

20. Nagata T, Kubota S, Watanabe I, Aoshima S. Chromatic aberration in pseudophakic eyes. J Jpn Ophthalmol Soc. 1999;103:237-242.

21. Negishi K, Ohnuma K, Hirayama N, Noda T. Effect of chromatic aberration on contrast sensitivity in pseudophakic eyes. Arch Ophthalmol. 2001;119:1154-1158.

22. Artal P, Manzanera S, Piers P, Weeber H. Visual effect of the combined correction of spherical and longitudinal chromatic aberrations. Opt Express. 2010;18:1637-1648.

23. Weeber HA, Piers PA. Theoretical performance of intraocular lenses correcting both spherical and chromatic aberration. $J$ Refract Surg. 2012;28:48-52.

24. Siedlecki D, Ginis HS. On the longitudinal chromatic aberration of the intraocular lenses. Optom Vis Sci. 2007;84: 984-989.

25. Bobier CW, Sivak JG. Chromoretinoscopy. Vision Res. 1978;18: 247-250.

26. Navarro R, Moreno E, Dorronsoro C. Monochromatic aberrations and point-spread functions of the human eye across the visual field. J Opt Soc Am A. 1998;15:2522-2529.

27. Moreno-Barriuso E, Lloves JM, Marcos S, Navarro R, Llorente L, Barbero S. Ocular aberrations before and after myopic corneal refractive surgery: LASIK-induced changes measured with laser ray tracing. Invest Ophthalmol Vis Sci. 2001;42:13961403.

28. Thibos LN, Wheeler W, Horner D. Power vectors: an application of Fourier analysis to the description and statistical analysis of refractive error. Optom Vis Sci. 1997; 74:367-375.

29. Thibos LN, Hong X, Bradley A, Applegate RA. Accuracy and precision of objective refraction from wavefront aberrations. $J$ Vis. 2004;4:329-351.

30. Cheng X, Bradley A, Thibos LN. Predicting subjective judgment of best focus with objective image quality metrics. J Vis. 2004;4:310-321.

31. Guirao A, Williams DR. A method to predict refractive errors from wave aberration data. Optom Vis Sci. 2003;80: 36-42.

32. Marcos S, Sawides L, Gambra E, Dorronsoro C. Influence of adaptive-optics ocular aberration correction on visual acuity at different luminances and contrast polarities. J Vis. 2008;8: $1-12$. 
33. Zhang XX, Bradley A, Thibos LN. Achromatazing the human eye: the problem of chromatic parallax. J Opt Soc Am A. 1991; 8:686-691.

34. Artal P, Guirao A. Contributions of the cornea and the lens to the aberrations of the human eyes. Opt Lett. 1998;23:1713-1715.

35. Barbero S, Marcos S, Merayo-Lloves J. Corneal and total optical aberrations in a unilateral aphakic patient. J Cataract Refract Surg. 2002;28:1594-1600.

36. Kruger PB, Pola J. Stimuli for accommodation: blur chromatic aberration and size. Vision Res. 1986;26:957-971.
37. Kruger PB, Mathews S, Aggarwala KR, Sanchez N. Chromatic aberration and ocular focus: Fincham revisited. Vision Res. 1993;33:1397-1411.

38. Thibos LN, Ye M, Zhang X, Bradley A. The chromatic eye: a new reduced-eye model of ocular chromatic aberration in humans. Appl Opt. 1992;31:3594-3600.

39. Harmening WM, Tiruveedhula P, Roorda A, Sincich LC. Measurement and correction of transverse chromatic offsets for multi-wavelength retinal microscopy in the living eye. Biomed Opt Express. 2012;3:2066-2077. 